\title{
Glucagon-like peptide-1 (GLP-1) receptor agonists and cardiovascular events in patients with type 2 diabetes mellitus: A meta-analysis of double-blind, randomized, placebo-controlled clinical trials
}

\author{
Jing Qin \\ Lanzhou University Second Hospital \\ Li Song ( $\square$ lisong1731@gmail.com) \\ Heidelberg University
}

Research article

Keywords: glucagon-like peptide-1, type 2 diabetes mellitus, cardiovascular events

Posted Date: October 31st, 2019

DOI: https://doi.org/10.21203/rs.2.16671/v1

License: (c) (i) This work is licensed under a Creative Commons Attribution 4.0 International License.

Read Full License 


\section{Abstract}

Background The cardiovascular effects of glucagon-like peptide-1 (GLP-1) receptor agonists are still controversial in the treatment of type 2 diabetes mellitus (T2DM) patients. The purpose of this study was to evaluate the risk of cardiovascular events of GLP-1 (albiglutide, exenatide, liraglutide and semaglutide) receptor agonists in T2DM patients.

Methods PubMed and Embase were searched to find relevant randomized controlled trials (RCTs) from inception to June 2019 that evaluated the effect of GLP-1 receptor agonists on cardiovascular events in patients with T2DM. The T2DM patients of all the eligible trials received either GLP-1 therapy or placebo, and the cardiovascular outcomes included death from cardiovascular causes, fatal or non-fatal myocardial infarction and fatal or non-fatal stroke.

Results We included 4 multinational double-blind randomized placebo-control trials that included a total of 36852 T2DM patients. The results indicated that GLP-1 receptor agonists reduced the risk of death from cardiovascular causes (RR: $0.86 ; 95 \% \mathrm{Cl}: 0.78-0.96 ; \mathrm{P}=0.005$ ), fatal or non-fatal myocardial infarction (RR: $0.85 ; 95 \% \mathrm{Cl}: 0.74-0.98 ; \mathrm{P}=0.03$ ) and fatal or non-fatal stroke (RR: $0.84 ; 95 \% \mathrm{Cl}$ : $0.75-$ $0.95 ; \mathrm{P}=0.007)$ compared with the placebo controls.

Conclusion We concluded that GLP-1 receptor agonist therapy had a protective cardiovascular effect compared with the placebo in the treatment of T2DM patients in trials with cardiovascular outcomes.

\section{Background}

Type 2 diabetes mellitus patients have a much higher risk of cardiovascular events, including death from cardiovascular causes, fatal or non-fatal myocardial infarction and fatal or non-fatal stroke [1]. The rates of cardiovascular death are 2- to 4-fold higher for patients with diabetes compared with the rates for those without diabetes [2]. GLP-1 receptor agonists, which are new glucose-lowering therapeutic agents in the treatment of type 2 diabetes mellitus, have been shown to affect the incidence of cardiovascular outcomes in patients with type 2 diabetes mellitus, although the results regarding GLP-1 receptor agonists remain inconsistent [3-4]. It is well known that GLP-1, as a peptide hormone, stimulates insulin secretion and inhibits glucagon secretion in a glucose-dependent manner [5]. An increasing number of studies have shown that glucagon-like peptide-1 (GLP-1) may improve endothelial functioning and may have direct effects in protecting the vascular system [6]. There are several GLP-1 receptor agonists that are used as therapeutic agents for treating type 2 diabetes mellitus patients in clinical fields. Recently, the GLP-1 receptor was believed to have an effect on individual cardiovascular outcomes in the treatment of diabetes, but not all GLP-1 receptor agonists showed the effect of reducing cardiovascular outcomes because of the varied effectiveness of the different GLP-1 drugs [3-4]. The use of GLP-1 receptor agonists to reduce cardiovascular outcomes in T2DM has become a new research and therapeutic area. Currently, no meta-analysis has concentrated on the long-term cardiovascular complications in GLP-1- 
treated T2DM patients. Previous studies have only focused on the short-term effects and safety of GLP1 receptor agonists on T2DM patients $[7,8]$.

Therefore, we performed a meta-analysis of double-blind randomized placebo-controlled clinical trials to investigate the cardiovascular complications of GLP-1 receptor agonists in T2DM patients. The cardiovascular outcomes included death from cardiovascular causes, fatal or non-fatal myocardial infarction and fatal or non-fatal stroke.

\section{Methods}

\section{Data sources and search strategy}

We comprehensively searched PubMed and Embase to find relevant randomized controlled trials (RCTs) from inception to June 2019 that evaluated the effect of GLP-1 receptor agonists on cardiovascular events in patients with T2DM. This meta-analysis was conducted and reported in accordance with the 2009 Preferred Reporting Items for Systematic Reviews and Meta-Analysis Statements[9]. The language was confined to English. The search terms were as follows: "incretin" OR "GLP-1" OR "glucagon-like peptide-1 analogue" OR "Liraglutide" OR "exenatide" OR "liraglutide" OR "lixisenatide" OR "albiglutide" OR "dulaglutide" OR "semaglutide" OR "taspoglutide" AND "type 2 diabetes mellitus" OR "T2DM" AND "randomized controlled trials" OR "RCT". We also comprehensively screened the references of reviews and articles in order to find more eligible articles.

Data selection criteria

The literature search was screened independently by two authors; if there were some inconsistencies, we discussed within the group until a consensus was reached. The research titles and abstracts were initially screened, and then we screened the study design, interventions, control, and outcomes in detail to determine the included trials.

The criteria for including eligible studies were as follows: (1) the studies were double-blind, randomized placebo-controlled trials; (2) the RCTs were evaluating GLP-1 versus placebo in T2DM patients; (3) there was a comparison of cardiovascular risk between GLP-1 receptor agonists and placebo in T2DM patients with or without cardiovascular diseases; and (4) a risk ratio (RR) with corresponding $95 \%$ confidence intervals (Cls) or data was reported.

Regarding the exclusion criteria, we excluded studies with the following criteria: (1) the case and control patients included other T2DM complications; (2) the controls received other conventional antidiabetic agents; and (3) duplicate publications, animal experimental studies, reviews, conference abstracts, or meta-analyses.

Data extraction and quality assessment 
The following data were extracted from the included RCTs by two authors independently: first author's name; publication year, country, sample size, study design, intervention, glycated haemoglobin, duration of diabetes and the follow-up periods. The quality of the included studies was assessed by the Cochrane Collaboration tool for the risk of bias [10]. The randomization, blinding, withdrawals, generation of random numbers, and concealment of allocation were essential components for each RCT. The quality of each results was assessed according to the Grading of Recommendations, Assessment, Development, and Evaluation (GRADE) system [11].

Data synthesis and statistical analysis

This meta-analysis was performed using Review Manager 5.3 software (RevMan), The Cochrane Collaboration, Copenhagen. For the cardiovascular events in our study, we calculated the the risk ratio (RR) with 95\% confidence intervals (Cls) to standardize the differences between the GLP-1 receptor agonist and placebo. The forest plots were conducted using a fixed-effect model if there was no obvious heterogeneity or using a random-effect model when heterogeneity of the included studies was obvious [12-13]. Additionally, the chi-squared $\left(\chi^{2}\right)$ test and the $I^{2}$ test were used to assess the heterogeneity between studies. When $P \leq 0.10$ and $I^{2}>50 \%$, the heterogeneity between those included studies was defined as obvious heterogeneity [14]. Moreover, if the $\mathrm{I}^{2}$ test value was $25-50 \%$, it was defined as mild heterogeneity, $50-75 \%$ as moderate heterogeneity, and $75 \%$ as severe heterogeneity. To measure publication bias, we performed a funnel plot and Egger's tests. A funnel plot was used to qualitatively measure the publication bias $[15,16]$, and $P \leq 0.05$ was considered significant publication bias in this meta-analysis.

\section{Results}

We obtained 1928 articles after searching PubMed and Embase from 2011 to June 2019. Then, we screened the titles and abstracts and removed the duplicate articles, reviews and conference abstracts, and 14 articles remained for evaluating the details of the full text to determine whether they met the inclusion criteria. Finally, 4 trials were included in this meta-analysis (Fig. 1). The Cochrane Collaboration tool was applied to evaluate the quality of the included trials. The results regarding the individual quality of the included trials are shown in Fig. 2.3.4.

The selected studies were published between 2016 and 2018. The GLP-1 receptor agonist arms included 18403 patients, and the placebo control arms included 18449 patients. The main characteristics of the included trials are presented in Table 1. The control treatment in the included trials was placebo according to the experimental trial treatment. The cardiovascular outcomes included death from cardiovascular causes, fatal or non-fatal myocardial infarction and fatal or non-fatal stroke.

There was no obvious heterogeneity in the four included studies $\left(I^{2}=0 \%\right.$, Cochran $Q$ test $\left.P=0.57\right)($ Fig. 2) regarding the risk of death from cardiovascular causes. Therefore, we used the fixed effect model in the RevMan software. GLP-1 receptor agonists reduced the risk of death from cardiovascular causes 
compared with the placebo (RR: $0.86 ; 95 \% \mathrm{Cl}: 0.78-0.96 ; \mathrm{P}=0.005)$ according to the results of the metaanalysis.

There was mild heterogeneity $\left(I^{2}=55 \%\right.$, Cochran $Q$ test $\left.P=0.08\right)(F i g .3)$ regarding the risk of fatal or nonfatal myocardial infarction in the included studies. Therefore, we used the random effect model in the RevMan software. The results showed that GLP-1 receptor agonists reduced the risk of fatal or non-fatal myocardial infarction compared with the placebo controls (RR: $0.85 ; 95 \% \mathrm{Cl}: 0.74-0.98 ; \mathrm{P}=0.03$ ).

There was also no evidence of heterogeneity observed across the included trials regarding fatal or nonfatal stroke $\left(I^{2}=0 \%\right.$, Cochran $Q$ test $\left.P=0.59\right)$ (see Fig. 4). The fixed effect model was applied in the RevMan software. The results of the meta-analysis indicated that GLP-1 receptor agonists reduced the risk of fatal or non-fatal stroke compared with the placebo (RR: $0.84 ; 95 \% \mathrm{Cl}: 0.75-0.95 ; \mathrm{P}=0.007$ ).

Because only four trials were included, which is less than ten, we have no evidence of publication bias in this meta-analysis by the funnel plot. No individual study had a significant effect on the pooled effect size according to the results of the sensitivity analysis at all end points.

\section{Discussion}

The objective of this meta-analysis was to explore the effect of GLP-1 receptor agonists on cardiovascular outcomes in type 2 diabetes mellitus patients. The results of this meta-analysis suggest that GLP-1 therapy has a significant impact on the incidence of death from cardiovascular causes, fatal or non-fatal myocardial infarction and fatal or non-fatal stroke in T2DM patients. There was no heterogeneity in these four included studies in their assessment of the effect of GLP-1 receptor agonists on the risk of death from cardiovascular causes and fatal or non-fatal stroke; however, there was mild heterogeneity regarding the risk of fatal or non-fatal myocardial infarction in the included studies, and the reasons may be the specific medicine molecule and GLP-1 receptor agonist dose tested, differences in the randomized patients (such as medical history and baseline characteristics), duration of follow-up years and adherence to treatment. As our findings are based on good-quality studies that were all multinational double-blind randomized placebo-control trials and our meta-analysis was based on a mean follow-up of 2.65 years (minimum 1.5 year - maximum 3.8 years), the confounding and attrition bias are controlled, so the risk of unreliable results is diminished.

Similar to our results, some studies $[17,18]$ also indicated that GLP-1 receptor agonists had a positive effect on the heart and enhanced cardiac function. GLP-1 receptor agonists have had cardiovascular protection effects in cardiovascular trials, which might be mediated through antiatherogenic mechanisms that contain direct and indirect effects on anti-inflammatory pathways, endothelial function and common cardiovascular risk factors (such as blood pressure) [19].

Some previous studies [20] found a lower incidence of cardiovascular disease (CVD) events when GLP-1 receptor agonists were compared with placebo. However, a comparison meta-analysis [21-23] indicated that there was no significant reduction in CVD events by GLP-1 receptor agonists. Similarly, Inzucchi et al. 
[24] believed that the evidence of GLP-1 receptor agonist cardiovascular protection was still limited, and the cardiovascular system benefits related to GLP-1 receptor agonists may be independent of its glucose, lipid, or energy metabolism effects.

The cardiovascular effects of GLP-1 receptor agonists might be mediated via antiatherogenic mechanisms that decrease cardiovascular risk over time. These mechanisms might include direct and indirect effects of GLP-1 receptor agonists on common cardiovascular risk factors (e.g., decreased blood pressure), anti-inflammatory pathways, cardiac output, ischaemic conditioning and endothelial function.

This meta-analysis has several strengths: (1) Only RCTs were included, so this meta-analysis eliminated the potential control group biases; (2) The large sample size of the 4 included trials allowed us to quantitatively evaluate the GLP-1 receptor agonist effects in T2DM patients; (3) A wide range of patient characteristics was represented, which ensured a comprehensive assessment of the effect of GLP-1 receptor agonists in the treatment of patients with T2DM.

This study has some limitations. First, the publication bias is an inevitable problem in any meta-analysis. Second, T2DM patients who received various GLP-1 receptor agonist drugs, such as albiglutide, exenatide, liraglutide and semaglutide, might have biased the meta-analysis results. Third, a more detailed analysis was restricted because the meta-analysis used pooled data.

\section{Conclusion}

The findings of this study indicated that GLP-1 receptor agonist therapy reduced the incidence of death from cardiovascular causes, fatal or non-fatal myocardial infarction and fatal or non-fatal stroke in the treatment of T2DM patients. We need additional large RCTs in the future to evaluate the treatment effects of GLP-1 receptor agonists in T2DM.

\section{Declarations}

Ethics approval and consent to participate: "Not applicable"

Consent for publication: "Not applicable"

Availability of data and materials: Data sharing is not applicable to this article as no datasets were generated or analyzed during the current study.

Competing interests: All authors declare that there are no conflict of interests or special relationships with industry in this meta-analysis.

Funding: "No funding" 
Authors' contributions: Li Song is the corresponding author of this study. She collected and analyzed data, wrote the manuscript and made the decision to submit and publish the manuscript.Jing Qin is the first author for the meta-analysis, including study design, collected and analyzed data, and wrote the manuscript.

Acknowledgements: "Not applicable"

\section{Abbreviations}

GLP-1 Glucagon-like peptide 1 receptor agonists

T2DM type 2 diabetes mellitus

RCTs randomized controlled trials

GRADE Grading of Recommendations, Assessment, Development, and Evaluation

CVD cardiovascular diseases

$\mathrm{RR}$ risk ratio

Cls confidence intervals

\section{References}

[1] Rawshani A, Rawshani A, Franzén S, et al. Mortality and cardiovascular disease in type 1 and type 2 diabetes.N Engl J Med 2017; 376: 1407-18.

[2] S.J. Haffner, H. Cassells, Hyperglycemia as a cardiovascular risk factor, Am. J. Me . 1013115 (Suppl. 8A) (2003) $6 S-11 S$.

[3] Pfeffer MA, Claggett B, Diaz R, et al. Lixisenatide in patients with type 2 diabetes and acute coronary syndrome. N Engl J Med 2015; 373: 2247-57.

[4] Holman RR, Bethel MA, Mentz RJ, et al. Effects of once-weekly exenatide on cardiovascular outcomes in type 2 diabetes. N Engl J Med 2017; 377: 1228-39.

[5]. Ussher JR, Drucker DJ. Cardiovascular biology of the incretin system. Endocrine Rev 2012; 33:187215 .

[6] A.J. Motta, J. Koska, P. Reaven, R.Q. Migrino, Vascular protective effects of diabetes medications that mimic or increase glucagon-like peptide-1 activity, Recent Pat. Cardiovasc. Drug Discov. 7 (2012) 2-9. 
[7] M. Monami, I. Dicembrini, C. Nardini, I. Fiordelli, E. Mannucci, Effects of glucagon-like peptide-1 receptor agonists on cardiovascular risk: a meta-analysis of randomized clinical trials, Diabetes Obes. Metab. 16 (2014) 38-47.

[8] M. Monami, F. Cremasco, C. Lamanna, et al., Glucagon-like peptide-1 receptor agonists and cardiovascular events: a meta-analysis of randomized clinical trials,Exp. Diabetes Res. 2011 (2011) 215764.

[9] D. Moher, A. Liberati, J. Tetzlaff, D.G. Altman, PRISMA Group, Preferred reporting items for systematic reviews and meta-analyses: the PRISMA statement, PLoS Med. 6 (2009), e1000097.

[10]. Higgins, J. P. et al. The Cochrane Collaboration's tool for assessing risk of bias in randomised trials. BMJ 343, d5928 (2011).

[11]. Guyatt, G. H. et al. Incorporating considerations of resources use into grading recommendations. Bmj 336, 1170-1173, https://doi.org/10.1136/bmj.39504.506319.80 (2008).

[12] Ades AE, Lu G, Higgins JP. The interpretation of random-effects meta-analysis in decision models. Med Decis Making 2005;25(6):646-54.

[13] DerSimonian R, Laird N. Meta-analysis in clinical trials revisited. Contemp Clin Trials 2015;45(Pt A):139-45.

[14] Higgins JP, Thompson SG, Deeks JJ, Altman DG. Measuring inconsistency in meta-analyses. BMJ 2003;327(7414):557-60.

[15] Egger M, Davey Smith G, Schneider M, Minder C. Bias in meta-analysis detected by a simple, graphical test. BMJ 1997;315(7109):629-34 [PubMed].

[16] Begg CB, Mazumdar M. Operating characteristics of a rank correlation test for publication bias. Biometrics 1994;50(4):1088-101 [PubMed].

[17] A.K. Bose, M.M. Mocanu, R.D. Carr, C.L. Brand, D.M. Yellon, Glucagon-like peptide 1 can directly protect the heart against ischemia/reperfusion injury, Diabetes 54 (2005) 146-151.

[18] T. Zhao, P. Parikh, S. Bhashyam, et al., Direct effects of glucagon-like peptide-1 on myocardial contractility and glucose uptake in normal and postischemic isolated rat hearts, J. Pharmacol. Exp. Ther. 317 (2006) 1106-1113.

[19] Nauck MA, Meier JJ, Cavender MA, Abd El Aziz M, Drucker DJ. 11 Cardiovascular actions and clinical outcomes with glucagon-like peptide- 1 receptor agonists and dipeptidyl peptidase-4 inhibitors. Circulation 2017; 136: 849-70. 
[20] F. Sun, K. Yu, S. Wu, et al., Cardiovascular safety and glycemic control of glucagon- like peptide-1 receptor agonists for type 2 diabetes mellitus: a pairwise and network meta-analysis, Diabetes Res. Clin. Pract. 98 (2012) 386-395.

[21] M. Monami, F. Cremasco, C. Lamanna, et al., Glucagon-like peptide-1 receptor agonists and cardiovascular events: a meta-analysis of randomized clinical trials,Exp. Diabetes Res. 2011 (2011) 215764.

[22] R.Ratner,J.Han,D.Nicewarner,I.Yushmanova,B.J.Hoogwerf,L.Shen,Cardiovascu- lar safety of exenatide BID: an integrated analysis from controlled clinical trials in participants with type 2 diabetes, Cardiovasc. Diabetol. 10 (2011) 22.

[23] S.P. Marso, J.B. Lindsey, J.M. Stolker, et al., Cardiovascular safety of liraglutide assessed in a patientlevel pooled analysis of phase 2:3 liraglutide clinical develop- ment studies, Diab. Vasc. Dis. Res. 8 (2011) 237-240.

[24] S.E. Inzucchi, D.K. McGuire, New drugs for the treatment of diabetes: part II: Incretin-based therapy and beyond, Circulation 117 (2008) 574-584.

\section{Figures}




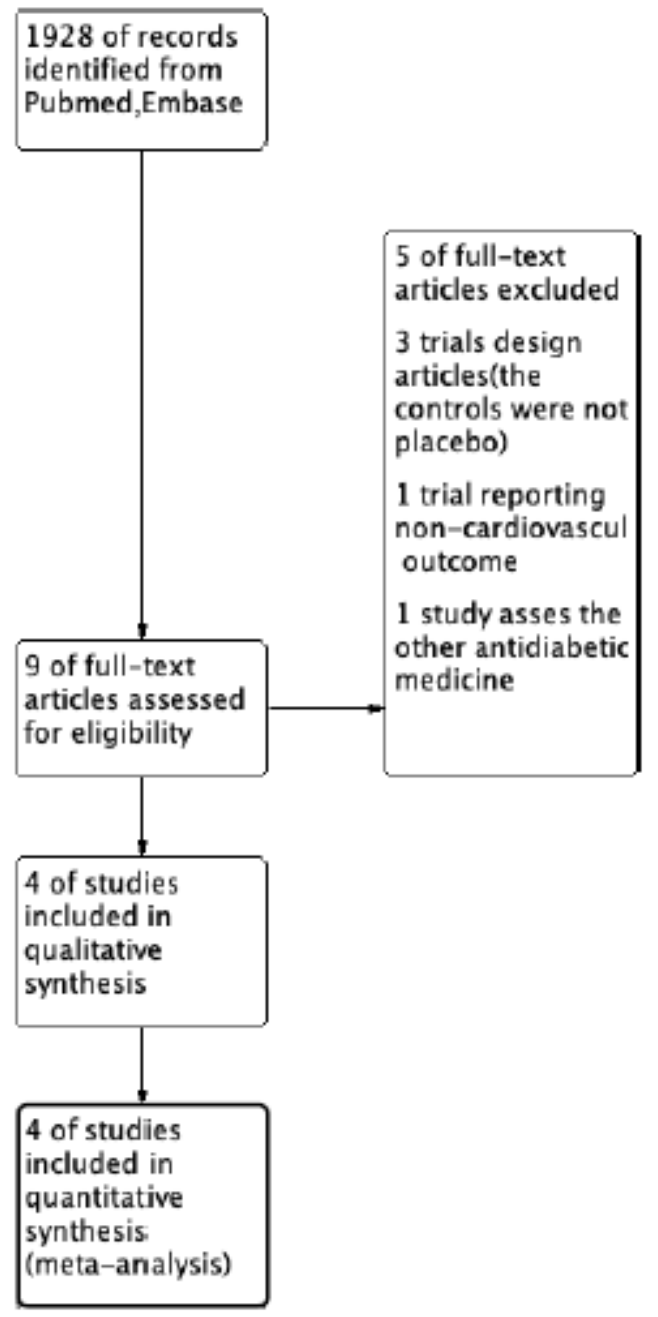

Figure 1

The flow diagram of the included studies

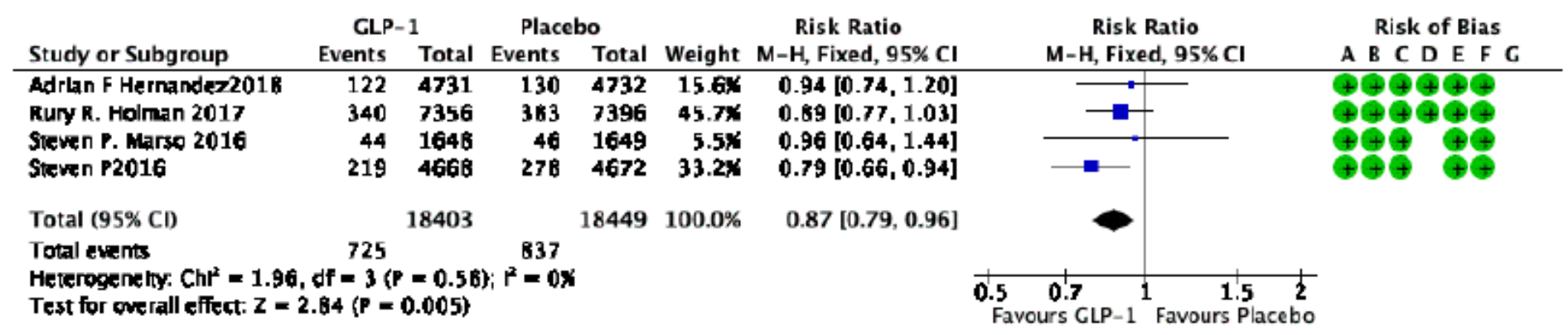

Risk of bias legend
(A) Random sequence generation (selection bias)
(B) Allocation concealment (selection bias)
(C) Blinding of participants and personnel (performance bias)
(D) Blinding of outcome assessment (detection bias)
(E) Incomplete outcome data (attrition bias)
(F) Selective reporting (reporting bias)
(G) Other bias

Figure 2 
Effect of GLP-1 versus placebo on death from cardiovascular causes.

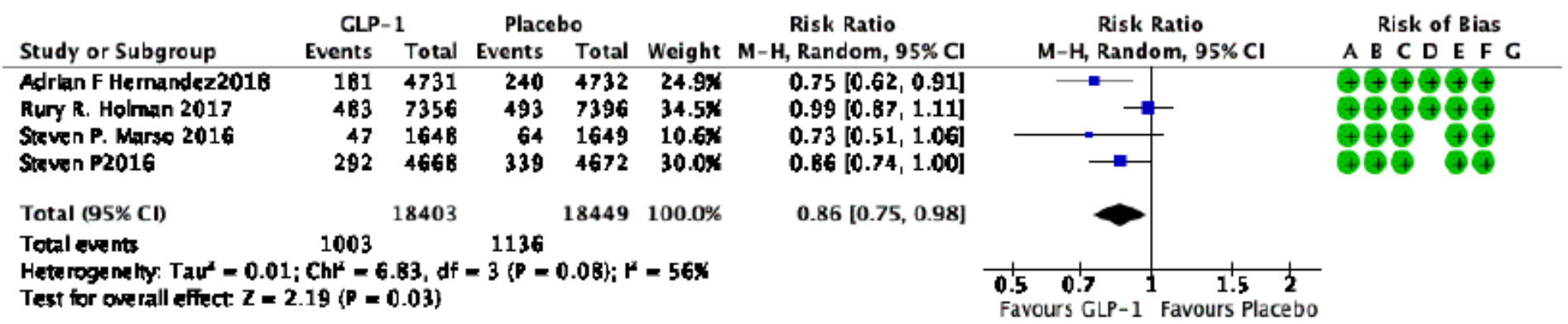

\section{Risk of bias legend}
(A) Random sequence generation (selection bias)
(B) Allocation concealment (selection bias)
(C) Blinding of participants and personnel (performance bias)
(D) Blinding of outcome assessment (detection blas)
(E) Incomplete outcome data (attrition blas)
(F) Selective reporting (reporting bias)
(G) Other bias

\section{Figure 3}

Effect of GLP-1 versus placebo on fatal or non-fatal myocardial infarction

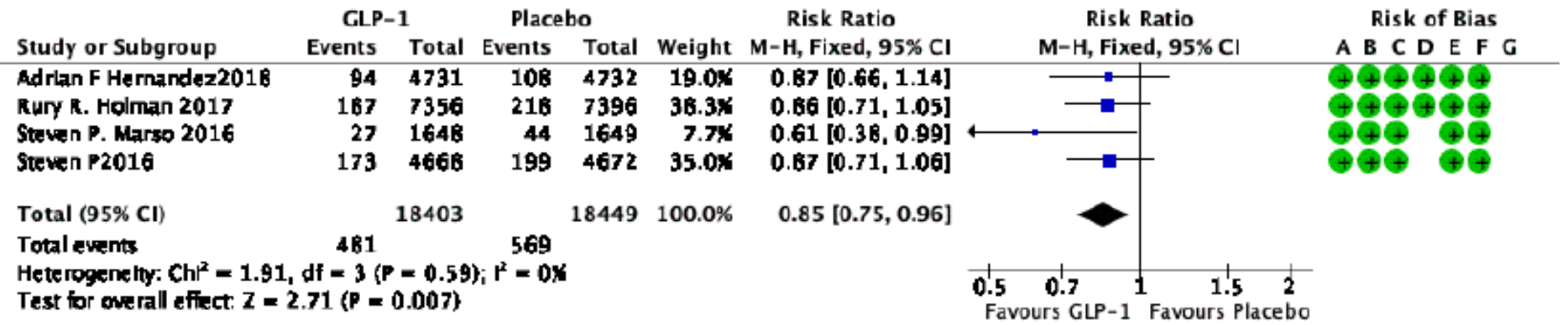

Risk of bias legend
(A) Random sequence generation (selection bias)
(B) Alocation concealment (selection bias)
(C) Blinding of participants and personnel (performance bias)
(D) Blinding of outcome assessment (detection bias)
(E) Incomplete outcome data (attrition bias)
(F) Selective reporting (reporting bias)
(C) Other bias

Figure 4

Effect of GLP-1 versus placebo on fatal or non-fatal stroke

\section{Supplementary Files}

This is a list of supplementary files associated with this preprint. Click to download.

- PRISMAchecklistforGLP1andCVD.docx 